\title{
Comparative Studies of Different Methods of Screening for Brown Plant Hopper (Nilaparvata lugens L.) Resistance in Rice Genotypes
}

\author{
Farzanakorabu*, B. Kisan, I. Shankergoud, Ayyangowda Patil, \\ Mahantesh Shivyoggaya and Sujayhurali
}
Department of Genetics and Plant Breeding, College of Agriculture, Raichur University of Agricultural Sciences, Raichur - 584 101, Karnataka, India

*Corresponding author

\begin{tabular}{l} 
K e y w o r d s \\
Brown planthopper, \\
Resistance, Rice \\
genotypes, \\
Screening methods \\
\hline Article Info \\
$\begin{array}{l}\text { Accepted: } \\
\text { 18 May } 2020 \\
\text { Available Online: } \\
\text { 10 June } 2020\end{array}$ \\
\hline
\end{tabular}

\begin{abstract}
A B S T R A C T
Among the plant hoppers, the brown planthopper (BPH) is a major threat to rice production and causes significant yield loss 25 per cent annually Nilaparvata lugens Stal. (BPH) is a typical phloem feeder. Both nymphs and adults suck sap from the leaves and leaf sheaths, which results in yellowing of leaves, reduced tillering, reduced plant height and increase in number of unfilled grains, whereas severe attack of BPH causes 'hopper burn' symptoms. It also transmits virus diseases like grassy stunt, ragged stunt and wilted stunt. Host-plant resistance is an effective environment friendly approach to reduce $\mathrm{BPH}$ damage and increase yield potential of cultivars. In this context in the present study an attempt was made to identify donors for BPH resistance by comparing three methods viz., field screening, honeydew test and nymphal survival test. By conducting field screening of 178 rice genotypes following standard evaluation system (IRRI, 2014), 17 genotypes were found to be resistant under field screening. These 17 genotypes were further used for conducting honey dew test and nymphal survival test in order to confirm the resistance which recorded only two and one resistant genotypes, respectively. During screening, BPT-5204 and PTB-33 were used as susceptible and resistant checks, respectively.
\end{abstract}

\section{Introduction}

More than 90 per cent of the world's rice is grown and consumed in Asia where 60 per cent of the global population lives. Globally rice is cultivated in an area of 163.2 million hectares with the production of 758.90 million tonnes, major cultivated area (134.52 million ha) is in Asia (Anon., 2017). Approximately 52 per cent of the global production of rice is lost annually owing to the damage caused by biotic stress, of which 25 per cent is attributed to the attack of insect pests. Among various biotic constraints of rice production, the insect 
pests are of prime importance and warm humid environment of the crop is also conducive for their survival and proliferation. Among the plant hoppers, the Brown planthopper ( $\mathrm{BPH}$ ) is a major threat to rice production and causes significant yield loss annually. Nilaparvata lugens Stal. is a typical phloem feeder. It also causes the reduction in chlorophyll, protein content of leaves and photosynthetic rate It is one of the most serious and destructive pests of rice throughout Asia. Both nymphs and adults suck sap from the leaves and leaf sheaths, which results in yellowing of leaves, reduced tillering, reduced plant height and increase in number of unfilled grains, whereas severe attack of BPH causes 'hopper burn' symptoms. It also transmits virus diseases like grassy stunt, ragged stunt and wilted stunt. Host-plant resistance is an effective environment friendly approach to reduce $\mathrm{BPH}$ damage and increase yield potential of cultivars therefore it is imperative to identify $\mathrm{BPH}$ resistance genes from diverse sources and incorporate them into rice cultivars. $\mathrm{BPH}$ populations on rice have been categorized in to four biotypes. The population in the east and Southeast Asia is reported as biotype 1, while biotype 2 originated in Indonesia and Vietnam as dominant biotype. Biotype 3 was produced in the laboratory at the International Rice Research Institute IRRI and in Japan, whereas biotype 4 is found only in South Asia. Historically, BPH was a minor pest of rice, emerged as a major pest in the tropical Asia during green revolution of the 1960s. In India, the first severe outbreak occurred in Kerala during 1973-74 destroying about 50,000 ha of rice. The severe outbreak of this pest was noticed during 2007 in parts of Cauvery command area in Karnataka and during 2008 in Haryana, Punjab and Delhi. The objective of the present study is to identify BPH resistance donors using field as well as controlled screening method.

\section{Materials and Methods}

\section{Plant material}

One hundred and seventy eight rice genotypes ranging from land races to improved lines were used for field screening against brown plant hopper (BPH) resistance (Table 1). These genotypes were obtained from the All India Coordinated Research Project Scheme, Agricultural Research Station, Gangavati, district Koppal Karnataka. The material was evaluated for resistance against BPH in field during kharif 2018 at the Agricultural Research Station, Gangavathi, University of Agricultural Sciences, Raichur. Gangavathi comes under northern dry zone of Karnataka with the annual rainfall of $523 \mathrm{~mm}$ which is one of hot spot region for BPH incidence. The material was screened under controlled conditions at ARS Gangavati during wet season of 2019. The screening methods included (i) Honeydew test and (ii) Nymphal survival test.

\section{Field screening}

All the 178 genotypes including two checks were sown on raised nursery beds with recommended cultural practices under unprotected conditions. Seedlings of 21days old were transplanted to main field with a spacing of $20 \mathrm{~cm}$ between rows and $15 \mathrm{~cm}$ between plants within a row was maintained. To maintain adequate BPH infestation, humid conditions were sustained by providing water level of $5 \mathrm{~cm}$ above the ground. Polythene sheet barrier of 2.5 feet height all around the planting area were erected as a barrier within 15 days. The resistant check used was PBT33 and susceptible check was BPT-5204. All around the border one meter length susceptible check (BPT-5204) was planted to build the maximum pest infestation. Scoring of genotypes was initiated when susceptible check shows hopper burn symptoms. Scoring 
was carried at two different stages of crop growth, one at 20 days after transplanting and other at 60 days after transplanting. Each entry was scored based on scoring system developed by the International Rice Research Institute, Philippines and each entry was scored as $0=$ no visible damage, $1=$ partial yellowing of first leaf, 3 = first and second leaves partially yellowing, $5=$ pronounced yellowing or some stunting, $7=$ mostly wilted plant but still alive, $9=$ the plant completely wilted or dead. Interpretation of results was based on standard evaluation system where the families with a mean rating of 0 to $3,3.1$ to 6.9 and 7 to 9 are designated as resistant, moderately resistant and susceptible, respectively.

\section{Honey dew test}

This test was carried out to screen genotypes for susceptibility and resistance by measuring feeding area of the leaves. All selected resistant genotypes were sown in pots during wet season 2019 under controlled condition (glass house). For each genotype, two pots were maintained containing five plants in each pot and 8-10 brown plant hopper adults were released at 40 days after transplanting. Varieties PTB-33 and BPT-5204 were also sown as checks along with all selected resistant genotypes. Many techniques have been developed to measure the feeding response of $N$. lugens on resistant and susceptible rice plants. The more important were the test of filter paper dipped in a solution of bromocresol green and the test of a Parafilm sachet. In the present study, the filter paper technique was used were honey dew collected on filter paper treated with bromocresol green by using following procedure. Ten plants were taken as reference to study the feeding activity. Filter papers were dipped with Bromocresol green solution. For each genotype five plants were tied with Bromocresol green dipped filter paper. The weight of the filter paper dipped in Bromocresol green was recorded. Brown plant hopper adults were released under controlled condition for feeding. After 24 hours, the weight of filter paper was recorded. Bromocresol green indicated phloem based honey dew as blue spots indicates resistance and susceptibility according to the amount of honey dew that appears on the filter paper. Change in the weight of filter paper indicates the feeding activity by BPH.

\section{Nymphal survival method}

The test was conducted to know the survival rate of the nymphs on different genotypes of rice. The numbers of surviving nymphs were recorded for all selected resistant genotypes and the two check varieties. Pot sowing was carried for each genotype under controlled condition (glass house). For each genotype two pots were maintained, each pot containing five plants were maintained. For each pot 20-25 nymphs were released on 50 days old transplanted rice plants for feeding. After 48 hrs of release of nymphs of insect, count was taken. More number of survivals of insect count indicates the genotype is susceptible and less number of insect count indicates the genotype is resistant.

\section{Results and Discussion}

\section{Field screening method}

In the present investigation 178 rice genotypes were screened during kharif 2018 for brown plant hopper resistance under field conditions at ARS Gangavathi and scoring was carried out at two growth stages of crop one was at 20 days and other was at 60 days after transplanting. Screening was done by 0-9 scale given by (IRRI, 2014). Scoring was carried after observing hopper burn symptoms on susceptible check, BPT 5204. 
Screening study at 20 days after transplanting reveals that out of 178 genotypes, 39 genotypes were found resistant with damage score between $0-1$. The 51 genotypes were found moderate resistant with damage score between 3-5. The 27 genotypes were found moderately susceptible with damage score between7-9 and 59 genotypes were found susceptible with the damage score of 9 .

In the second scoring at 60 days after transplanting out of 178 genotypes 17 genotypes were found resistant with damage score between $0-1$. The 32 genotypes were found moderate resistance with damage score between 3-5. The 127 genotypes were found susceptible with damage score between 7-9.

In the above both field screening study it was found that out of 178 genotypes only 17 genotypes were found to be resistant with damage score between $0-1$ as presented in the Table 2 viz., IR-58025A, IR-68888A, IR68897A IR- 80555A, Uggi, Karpoosalibudda, Atikayi, BA-4, BA-13, BA-15, BA-19, BA28, BA-3, BA-20, BA-17, BA-3 and BA-33 . These 17 genotypes were further used for rescreening for BPH tolerance using honey dew test and nymph survival test.

A similar trend was noticed to the results obtained by Akshay et al., (2011) who screened 57 accessions of rice, among them only seven genotypes showed resistant reaction, with a damage score of 1 . Rest of the entries were found to be susceptible with a damage score of 7 to 9. Bhogadhi and Bentur (2015) reported similar findings by evaluating 20 genotypes of rice resistant to brown plant hopper by conducting field screening.

Similar results were also reported by Saxena and Saxena (2009), Santhanalaksmi et al., (2010), Jhansi Lakshmi et al., (2010), Madurangi et al., (2010), Sable and Rana (2011), Yongfu et al., (2011), Youg et al.,
(2012), Ali et al., (2012), (2014), Bhogadhi and Bentur (2015), Varma et al., (2014), Shoukat and Pandey (2016) and Udayshree et al., (2018).

\section{Honey dew secretion test}

Seventeen genotypes were tested for honey dew secretion studies, more secretion of honey dew was observed in genotypes BA33 $(0.85)$ and BA17 (0.85) followed by BA20 (0.75) and least secretion was observed by resistant genotypes IR-68897A (0.52) and BA-31(0.51) The check PTB-33 showed least secretion (0.5) and other BPT-5204 check showed highest secretion.

The change in the weight of filter paper indicates amount of phloem based honeydew secreted. More secretion of honeydew and increase in the weight of filter paper after feeding indicates that the variety is susceptible to brown plant hopper. No or less change in the weight of filter paper, indicates that no or less feeding activity which indicates that the variety is resistant. Less change in the weight was observed in only two genotypes IR 68888A and BA-31 (Table 3).

These results correlate with the findings of Kale et al., (2007) who evaluated 121 promising rice genotypes for BPH reaction, among which three genotypes were found to be resistant and 20 were moderately resistant, whereas 98 genotypes were susceptible, the resistant rice genotype had the least feeding values $\left(28.8 \mathrm{hr} .39 \mathrm{~mm}^{2}\right.$ honeydew secretion area) with the highest number of probes (25 to 40 per seedling in $24 \mathrm{hrs}$ ) and feeding duration with vice versa in susceptible rice genotypes. Similar results were also reported by Yongfu et al., (2011) and Bhogadhi and Bentur (2015) who evaluated genotypes by conducting honeydew secretion and found resistant varieties. 
Table.1 Brown plant hopper reaction of paddy genotypes at 20 days and 60 days after transplanting at ARS, Gangavathi

\begin{tabular}{|c|c|c|c|c|c|c|c|c|c|c|c|}
\hline \multirow[t]{2}{*}{ Sl. } & \multirow[t]{2}{*}{ Genotypes } & \multirow{2}{*}{$\begin{array}{l}\text { DS } \\
\text { at }\end{array}$} & \multirow[t]{2}{*}{ FR } & \multirow[t]{2}{*}{ DS at } & \multirow[t]{2}{*}{ FR } & 45 & Pattera & 5 & MR & 9 & HS \\
\hline & & & & & & 46 & Atikayi & 1 & $\mathrm{R}$ & 1 & $\mathrm{R}$ \\
\hline \multirow[t]{2}{*}{ No. } & & \multirow{2}{*}{$\begin{array}{l}20 \\
\mathrm{DA} \\
\mathrm{T}\end{array}$} & & \multirow{2}{*}{$\begin{array}{l}60 \\
\text { DAT }\end{array}$} & & 47 & Raichursanna & 6 & MR & 9 & HS \\
\hline & & & & & & 48 & Champa & 5 & MR & 9 & HS \\
\hline 1 & IR-58025A & 0 & $\mathrm{R}$ & 0 & $\mathrm{R}$ & 49 & Sindhumati & 9 & S & 9 & HS \\
\hline 2 & IR-68888A & 0 & $\mathrm{R}$ & 1 & $\mathrm{R}$ & 50 & Kittachudi & 9 & S & 9 & HS \\
\hline 3 & IR-68897A & 0 & $\mathrm{R}$ & 0 & $\mathrm{R}$ & 51 & Aknalu & 7 & MS & 9 & HS \\
\hline 4 & IR-80555A & 0 & $\mathrm{R}$ & 1 & $\mathrm{R}$ & 52 & Lanka-1 & 9 & S & 9 & HS \\
\hline 5 & IR-80561A & 0 & $\mathrm{R}$ & 9 & HS & 53 & Gini sali & 9 & $\mathrm{~S}$ & 9 & HS \\
\hline 6 & IR-58025A & 0 & $\mathrm{R}$ & 4 & MR & 54 & Doddabairanelli & 6 & MR & 9 & HS \\
\hline 7 & IR-68888A & 0 & $\mathrm{R}$ & 5 & MR & 55 & Kempottabhatta & 6 & MR & 9 & HS \\
\hline 8 & IR-68897A & 0 & $\mathrm{R}$ & 9 & HS & 56 & Deepakrani & 6 & MR & 9 & HS \\
\hline 9 & IR-80555A & 0 & $\mathrm{R}$ & 4 & MR & 57 & Daserapachi & 9 & $\mathrm{~S}$ & 9 & HS \\
\hline 10 & IR-79156A & 0 & $\mathrm{R}$ & 8 & S & 58 & Mukkanna & 9 & $\mathrm{~S}$ & 9 & HS \\
\hline 11 & IR-58025B & 0 & $\mathrm{R}$ & 8 & S & 59 & Rani & 5 & MR & 9 & HS \\
\hline 12 & IR-68888B & 0 & $\mathrm{R}$ & 9 & HS & 60 & Shakti & 5 & MR & 9 & HS \\
\hline 13 & IR-68897B & 0 & $\mathrm{R}$ & 9 & HS & 61 & Ellisali & 9 & $\mathrm{~S}$ & 9 & HS \\
\hline 14 & IR-80555B & 0 & $\mathrm{R}$ & 9 & HS & 62 & Bhogyalaya & 9 & $\mathrm{~S}$ & 9 & HS \\
\hline 15 & IR-80561B & 0 & $\mathrm{R}$ & 4 & MR & 63 & Doddattakal & 9 & $\mathrm{~S}$ & 9 & HS \\
\hline 16 & IR-58025B & 0 & $\mathrm{R}$ & 9 & HS & 64 & IET-22110 & 9 & S & 9 & HS \\
\hline 17 & IR-68888B & 0 & $\mathrm{R}$ & 9 & HS & 65 & Arunnara vadlu & 9 & $\mathrm{~S}$ & 9 & HS \\
\hline 18 & IR-68897B & 0 & $\mathrm{R}$ & 9 & HS & 66 & Kalakolla & 3 & MR & 9 & HS \\
\hline 19 & IR-80555B & 0 & $\mathrm{R}$ & 9 & HS & 67 & Karikaswati & 5 & MR & 9 & HS \\
\hline 20 & IR-79156B & 0 & $\mathrm{R}$ & 9 & $\mathrm{HS}$ & 68 & Salem sanna & 7 & MS & 9 & HS \\
\hline 21 & Rajavani & 0 & $\mathrm{R}$ & 9 & $\frac{\mathrm{HS}}{\mathrm{HS}}$ & 69 & Matilaga & 9 & $\mathrm{~S}$ & 9 & HS \\
\hline 22 & Kaviraja & 0 & $\mathrm{R}$ & 0 & HS & 70 & Massura & 9 & $\mathrm{~S}$ & 9 & HS \\
\hline 23 & Toimallli & 0 & $\mathrm{R}$ & 0 & HS & 71 & HMT nellu & 9 & S & 9 & HS \\
\hline 24 & Tulsibasha & 0 & & 9 & HS & 72 & Badashabhog & 7 & $\mathrm{~S}$ & 9 & HS \\
\hline 25 & Uggi & 0 & & 5 & MR & 73 & Meesebatta & 9 & $\mathrm{~S}$ & 9 & HS \\
\hline 26 & Jeerigisamba & 0 & $R^{K}$ & 2 & $\mathrm{R}$ & 74 & Kumkumsali & 9 & S & 9 & HS \\
\hline 27 & Adeppa & 5 & & 9 & HS & 75 & Khagisuli & 9 & S & 9 & HS \\
\hline 28 & Jayaganmanji & 3 & & 9 & HS & 76 & Ummata & 9 & S & 9 & HS \\
\hline 29 & Karpoosalibudda & 1 & & 9 & HS & 77 & Rajkumar & 9 & $\mathrm{~S}$ & 9 & HS \\
\hline 30 & lanka-2 & 3 & & 1 & 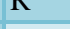 & 78 & Karımundaga & 7 & MS & 9 & HS \\
\hline 31 & Mahadi & 9 & & & III) & 19 & Sannarajakayam & 5 & MR & 9 & HS \\
\hline 32 & & & & & & 80 & Doddigya & 1 & $S$ & 9 & HS \\
\hline 33 & & & & 3 & HS & 81 & Kanada tumba & 7 & $\mathrm{~S}$ & 9 & HS \\
\hline \multirow{2}{*}{34} & Bilimundaga & 5 & MR & 5 & MR & 82 & Athikaraya & 9 & $\mathrm{~S}$ & 9 & HS \\
\hline & \multirow{2}{*}{$\begin{array}{l}\text { Kempukoorigune } \\
\text { llu }\end{array}$} & \multirow{2}{*}{9} & \multirow[t]{2}{*}{$\mathrm{S}$} & \multirow[t]{2}{*}{9} & \multirow[t]{2}{*}{ HS } & 83 & Krishnakanda & 9 & S & 9 & HS \\
\hline 35 & & & & & & 84 & Jodumuni & 7 & $\mathrm{~S}$ & 9 & HS \\
\hline 36 & Kododdi & & S & 9 & HS & 85 & Champakali & 7 & $\mathrm{~S}$ & 9 & HS \\
\hline 37 & Intan & & S & 5 & $S$ & 86 & Madrassanna & 9 & $\mathrm{~S}$ & 9 & HS \\
\hline 38 & Marabatta & 7 & MS & 9 & HS & 87 & Siddhasanna & 7 & S & 9 & HS \\
\hline 39 & Kamadari & 9 & $\mathrm{~S}$ & 9 & HS & 88 & Helaga & 9 & $\mathrm{~S}$ & 9 & HS \\
\hline 40 & Gandhasale & 9 & S & 9 & HS & 89 & Chindonni & 7 & MS & 9 & HS \\
\hline 41 & Kempujaddu & 5 & MR & 9 & HS & 90 & Kumuda & 9 & $\mathrm{~S}$ & 9 & HS \\
\hline 42 & Marayyabudda & 7 & MS & 9 & HS & 91 & Hunasehoovinabatt & 5 & MR & 9 & HS \\
\hline 43 & $\begin{array}{l}\text { Kyasari } \\
\text { Karihegga }\end{array}$ & $\begin{array}{l}7 \\
3\end{array}$ & MS & 9 & HS & 92 & Parimalasanna & 7 & MS & 9 & HS \\
\hline 44 & Rangoli & 5 & MR & 9 & HS & 93 & H. varanasi & 9 & $\mathrm{~S}$ & 9 & HS \\
\hline & & & & & & 94 & Gubugunda & 3 & MR & 9 & HS \\
\hline
\end{tabular}


Int.J.Curr.Microbiol.App.Sci (2020) 9(6): 1914-1922

\begin{tabular}{|c|c|c|c|c|c|c|c|c|c|c|c|}
\hline 95 & Padmarekha & 3 & MR & 4 & $\mathrm{~S}$ & 144 & GNV-1108 & 7 & MS & 9 & HS \\
\hline 96 & Redshalini & 3 & MR & 9 & HS & 145 & GNN-16-02 & 3 & MR & 5 & MR \\
\hline 97 & Kadalikenda & 3 & MR & 5 & MR & 146 & RNR-15048 & 5 & MR & 5 & MR \\
\hline 98 & Navali & 5 & MR & 5 & MR & 147 & IET-25497 & 9 & $\mathrm{~S}$ & 9 & HS \\
\hline 99 & Mapalasamba & 7 & MS & 9 & HS & 148 & $6502 \mathrm{~K}-17$ & 7 & MS & 9 & HS \\
\hline 100 & Dappavalye & 3 & MR & 5 & MR & & (Mutant) & & & & \\
\hline 101 & Bangarasanna & 9 & $\mathrm{~S}$ & 9 & HS & 149 & GNV-1109 & 0 & $\mathrm{R}$ & 5 & MR \\
\hline 102 & Pusa-5211 & 9 & S & 9 & HS & 150 & GNV-10-89 (C) & 5 & MR & 6 & MR \\
\hline 103 & Narikela & 9 & S & 9 & HS & 151 & MTU-1010 (C) & 5 & MR & 5 & MR \\
\hline 104 & Bheemasale & 9 & $\mathrm{~S}$ & 9 & HS & 152 & BPT-mutant 601- & 5 & MR & 5 & MR \\
\hline 105 & Kayam & 9 & $\mathrm{~S}$ & 9 & HS & & 1 & & & & \\
\hline 106 & Navara & 9 & S & 9 & HS & 153 & BPT-mutant 603- & 5 & MR & 6 & MR \\
\hline 107 & Baguri & 3 & MR & 9 & HS & & & & & & \\
\hline 108 & NM-5-2 & 9 & $\mathrm{~S}$ & 9 & HS & 154 & BPT-mutant 609 & 7 & MS & 9 & HS \\
\hline 109 & Gulwadisanna & 9 & S & 9 & HS & 155 & RP-Bio-226 mutant & & 5 & MR & 5 \\
\hline 110 & Meternellu & 5 & MR & 9 & HS & & $614-1$ & & & & \\
\hline 111 & Satva & 7 & MS & 9 & HS & MR & & & & & \\
\hline 112 & Nettabatta & 5 & MR & 9 & HS & 156 & RP-Bio-226 mutant & 615 & 5 & MR & 5 \\
\hline 113 & Kaviraja-1 & 9 & $\mathrm{~S}$ & 9 & HS & MR & & & & & \\
\hline 114 & Toimalli-1 & 9 & S & 9 & HS & 157 & RP-Bio-226 mutan & 618 & 7 & MS & 9 \\
\hline & & & & & & HS & & & & & \\
\hline 115 & Jeregesamba & 9 & S & 9 & HS & 158 & $\begin{array}{l}\text { BPT-mutant } \\
626\end{array}$ & 5 & MR & 6 & MR \\
\hline 116 & Imtan-1 & 7 & MS & 9 & HS & 159 & BPT-mutant & 7 & MS & 9 & HS \\
\hline 117 & Kamadhari-1 & 7 & MS & 9 & HS & & 633 & & & & \\
\hline 118 & Kyasari-1 & 7 & MS & 9 & HS & 160 & BPT-mutant & 7 & MS & 7 & $S$ \\
\hline 119 & Kempottebatta & 7 & MS & 9 & HS & & 638 & & & & \\
\hline 120 & Deepakrani-1 & 9 & S & 9 & HS & 161 & BPT-mutant & 7 & MS & 9 & HS \\
\hline 121 & Masura-1 & 9 & S & 9 & HS & & 619 & & & & \\
\hline 122 & Ummati-1 & 9 & $\mathrm{~S}$ & 9 & HS & 162 & BPT-mutant & 5 & MR & 6 & MR \\
\hline 123 & Doddigya-1 & 7 & MS & 9 & HS & & 653 & & & & \\
\hline 124 & Kumoda-1 & 9 & $\mathrm{~S}$ & 9 & HS & 103 & $\begin{array}{l}\text { BPI-mutant } \\
1807\end{array}$ & 9 & $\mathrm{~s}$ & 9 & \\
\hline 125 & H.Varanasi-1 & 9 & S & 9 & HS & 164 & RP-Bio-226 & 7 & MS & 7 & S \\
\hline 126 & Gulwadisanna-1 & 5 & MR & 9 & HS & 165 & Gangavati & 7 & MS & 9 & HS \\
\hline 127 & HMT sona & 7 & MS & 9 & HS & & sona & & & & \\
\hline 128 & KRH-4 & 4 & MR & 5 & MR & 166 & CSR-22 & 7 & MS & 9 & HS \\
\hline 129 & BPT-mutant & 3 & MR & 5 & MR & 167 & BA-4 & 1 & $\mathrm{R}$ & 1 & $\mathrm{R}$ \\
\hline 130 & BPT-mutant 603 & 5 & MR & 5 & MR & 168 & BA-13 & 1 & $\mathrm{R}$ & 1 & $\mathrm{R}$ \\
\hline 131 & YPT-M-Gontra & 5 & MR & 5 & MR & 169 & BA-15 & 0 & $\mathrm{R}$ & 0 & $\mathrm{R}$ \\
\hline & Bidhan-3 & & & & & 170 & BA-19 & 1 & $\mathrm{R}$ & 1 & $\mathrm{R}$ \\
\hline 132 & RP-Bio-226 & 5 & MR & 5 & MR & 171 & BA-28 & 0 & $\mathrm{R}$ & 1 & $\mathrm{R}$ \\
\hline & mutant 614 & & & & & 172 & BA-3 & 0 & $\mathrm{R}$ & 1 & $\mathrm{R}$ \\
\hline 133 & IET-25451 & 3 & MR & 5 & MR & 173 & BA-20 & 0 & $\mathrm{R}$ & 1 & $\mathrm{R}$ \\
\hline 134 & IET-27162 & 3 & MR & 9 & HS & 174 & BA-17 & 0 & $\mathrm{R}$ & 0 & $\mathrm{R}$ \\
\hline 135 & IET-25520 & 5 & MR & 5 & MR & 175 & BA-3 & 0 & $\mathrm{R}$ & 1 & $\mathrm{R}$ \\
\hline 136 & SWW-09-32-IL & 3 & MR & 4 & MR & 176 & BA-33 & 0 & $\mathrm{R}$ & 1 & $\mathrm{R}$ \\
\hline 137 & RP-Bio-226 & 9 & $\mathrm{~S}$ & 9 & HS & 177 & PTB-33 (RC) & 0 & $\mathrm{R}$ & 0 & $\mathrm{R}$ \\
\hline & (Improved BPT) & & & & & 178 & BPT-5204 (SC) & 9 & $\mathrm{~S}$ & 9 & HS \\
\hline 138 & BPT-mutant 633 & 5 & MR & 6 & MR & \multirow{6}{*}{\multicolumn{6}{|c|}{$\begin{array}{l}\text { DS- Damage Score, R-Resistant, MR-Moderately Resistant, } \\
\text { MS-Moderately susceptible, MR-Moderately Resistant, HS- } \\
\text { Highly susceptible, S-Susceptible, FR- Reaction of BPH in } \\
\text { field, DAT- Days after transplanting, RC- Resistant check, } \\
\text { SC- Susceptible check }\end{array}$}} \\
\hline 139 & $\begin{array}{l}\text { Gangavati sona } \\
\text { (C) }\end{array}$ & 5 & MR & 5 & MR & & & & & & \\
\hline 140 & IABT-17 & 9 & S & 9 & HS & & & & & & \\
\hline 141 & Gangavati sanna & 9 & $\mathrm{~S}$ & 9 & HS & & & & & & \\
\hline 142 & IET-22066 & 3 & MR & 6 & MR & & & & & & \\
\hline 143 & IET-24767 & 7 & MS & 7 & S & & & & & & \\
\hline
\end{tabular}


Int.J.Curr.Microbiol.App.Sci (2020) 9(6): 1914-1922

Table.2 Selected 17 genotypes showing resistance at 20 days and 60 days after transplanting

\begin{tabular}{|l|l|l|l|l|l|}
\hline Sl. & Genotypes name & DS at & FR & DS at & FR \\
\hline N & & $\begin{array}{l}20 \\
\text { o. }\end{array}$ & & 60 & \\
\hline 1 & IR 58025A & 0 & R & 0 & R \\
\hline 2 & IR 68888A & 0 & R & 1 & R \\
\hline 3 & IR 68897A & 0 & R & 0 & R \\
\hline 4 & IR 80555A & 0 & R & 1 & R \\
\hline 5 & Uggi & 0 & R & 2 & R \\
\hline 6 & Karpoosalibudda & 1 & R & 1 & R \\
\hline 7 & Atikayi & 1 & R & 1 & R \\
\hline 8 & BA-4 & 1 & R & 1 & R \\
\hline 9 & BA-13 & 1 & R & 1 & R \\
\hline 10 & BA-15 & 1 & R & 0 & R \\
\hline 11 & BA-19 & 0 & R & 1 & R \\
\hline 12 & BA-28 & 0 & R & 1 & R \\
\hline 13 & BA-3 & 0 & R & 1 & R \\
\hline 14 & BA-20 & 0 & R & 0 & R \\
\hline 15 & BA-17 & 0 & R & 1 & R \\
\hline 16 & BA-30 & R & 1 & R \\
\hline 17 & B1A-33 & 0 & & 1 & \\
\hline
\end{tabular}

DS- Damage Score, FR- Reaction of BPH in field, DAT- Days after transplanting

Table.3 Screening of selected genotypes by honey dew secretion test method

\begin{tabular}{|c|c|c|c|c|}
\hline SL. & Genotypes & \multirow{2}{*}{\multicolumn{3}{|c|}{$\begin{array}{l}\text { Wt. of filter Wt. of filter Final wt. - } \\
\text { paper before paper after Initial wt. }\end{array}$}} \\
\hline \multirow[t]{2}{*}{ No. } & & & & \\
\hline & & $\begin{array}{l}\text { feeding } \\
(\mathrm{g})\end{array}$ & $\begin{array}{l}\text { feeding } \\
\text { (g.) }\end{array}$ & \\
\hline 1 & IR 58025A & 0.50 & 0.55 & 0.05 \\
\hline 2 & IR 68888A & 0.50 & 0.51 & 0.01 \\
\hline 3 & IR 68897A & 0.50 & 0.59 & 0.09 \\
\hline 4 & IR $80555 A$ & 0.50 & 0.58 & 0.08 \\
\hline 5 & $\begin{array}{l}\text { Karpoosalibud } \\
\text { da }\end{array}$ & 0.50 & 0.59 & 0.09 \\
\hline 6 & Atikayi & 0.50 & 0.59 & 0.09 \\
\hline 7 & IR 68897B & 0.50 & 0.52 & 0.02 \\
\hline 8 & BA-03 & 0.50 & 0.65 & 0.15 \\
\hline 9 & BA-20 & 0.50 & 0.75 & 0.25 \\
\hline 10 & BA-17 & 0.50 & 0.85 & 0.35 \\
\hline 11 & BA-33 & 0.50 & 0.85 & 0.35 \\
\hline 12 & BA-28 & 0.50 & 0.70 & 0.20 \\
\hline 13 & BA-3 & 0.50 & 0.71 & 0.21 \\
\hline 14 & BA-31 & 0.50 & 0.51 & 0.01 \\
\hline 15 & BA-4 & 0.50 & 0.62 & 0.12 \\
\hline 16 & BA-15 & 0.50 & 0.59 & 0.09 \\
\hline 17 & BA-19 & 0.50 & 0.62 & 0.12 \\
\hline Che & PTB-33 (RC) & 0.50 & 0.50 & 0.00 \\
\hline & BPT-5204 (SC) & 0.50 & 0.92 & 0.42 \\
\hline
\end{tabular}

RC- Resistant check, SC- Susceptible check 
Table.4 Screening of selected genotypes by nymphal survival test method

\begin{tabular}{|l|l|l|}
\hline $\begin{array}{l}\text { Sl. } \\
\text { No. }\end{array}$ & Genotypes & $\begin{array}{l}\text { Nymphal } \\
\text { survival } \\
\text { ( out of 20-25) }\end{array}$ \\
\hline 1 & IR 58025A & 21 \\
\hline 2 & IR 6888A & 30 \\
\hline 3 & IR 68897A & 11 \\
\hline 4 & IR 80555A & 12 \\
\hline 5 & Uggi & 14 \\
\hline 6 & Karpoosalibudda & 15 \\
\hline 7 & Atikayi & 2 \\
\hline 8 & BA-03 & 15 \\
\hline 9 & BA-20 & 16 \\
\hline 10 & BA-28 & 17 \\
\hline 11 & BA-13 & 14 \\
\hline 12 & BA-17 & 13 \\
\hline 13 & BA-30 & 12 \\
\hline 14 & BA-33 & 11 \\
\hline 15 & BA-4 & 12 \\
\hline 16 & BA-15 & 3 \\
\hline 17 & BA-19 & 11 \\
\hline 18 & PTB-33 (RC) & 55 \\
\hline 19 & BPT-5204 (SC) & \\
\hline & RC- Resistant check, SC- Susceptible check \\
\hline
\end{tabular}

\section{Nymphal survival method}

Wide range of variation was noticed in nymphal survival method with respect to nymphal survival rate as it varied from 2.00 to 30.00 with the average of 13.21. The genotype, Atikayi (2.00) and BA-19 (3) showed least nymph survival rate among resistant genotypes (Table 4).

A similar trend was noticed in the results obtained by Venugopala Reddy and Kalode (1985) who screened a total of 1070 rice varieties to identify better sources of resistance to brown plant hopper, mainly from Assam rice collection. Further, in mass screening replicated tests 17 varieties were identified as resistant and73 varieties were moderately resistant. Similar results were also obtained by Sable and Rana (2011) and Bhogadhi and Bentur (2015) who screened rice genotypes under controlled glass house conditions by conducting nymphal survival test.
It was found from the above different methods of screening that field screening method was better as it was also followed by many researchers as compared to honeydew secretion test and nymphal survival test to screen the genotypes against BPH. Seventeen resistant genotypes were obtained from 178 rice genotypes by field screening method as compared to honeydew secretion test and nymphal survival test where only two and one resistant genotypes, respectively were obtained.

\section{References}

Akshay M C, 2011, Studies on mechanism of resistance to brown planthopper, Nilaparvata lugens (Stal.) in rice. M.Sc. (Agri.) Thesis, University of Agricultural Sciences, Bengaluru, Karnataka, India.

Ali M P, Salem S A, Begum A M, Anwaruddin A B M, Alam M Z and Dingcheng Huang, 2012, Screening of rice genotypes for resistance to the brown Planthopper, 
Nilaparvata lugen Stal. Cereal Research Communications, 40(4), 502-508.

Anonymous, 2017, Rice Market Monitor. FAO, 20(1): 1-38.

Bhogadhi S C and Bentur J S, 2015, Screening of rice genotypes for resistance to brown plant hopper biotype 4 and detection of $\mathrm{BPH}$ resistance genes. International Journal of Life Sciences, Biotechnology and Pharma Research, 4: 90-95.

Jhansi Lakshmi V, Mallikarjuna Swamy B P, Kaladhar K and Sarla N, 2010, BPH resistance introgression lines of Swarna/ Oryza nivara and KMR3/O.rufipogan. Directorate of Rice Research Newsletter, 8(4): 4.

Kale G B, Karnewa S D and Dudhare M S, 2007, Evaluation of rice accessions for mechanism of resistance to brown plant hopper (Nilaparvata lugens Stal.). New Botanists, 34(1): 121-126.

Madurangi S A P, Ratnasekera D, Hemachandra P V and Senanayake S G J N, 2010, Evaluation of brown planthopper Nilaparvata lugens (Stal.) resistance in Oryza nivara wild rice accessions found in Sri Lanka. Proceedings of the $15^{\text {th }}$ International Forestry and Environment Symposium. Department of Forestry and Environmental Science, University of Sri Jayewardenepura, Sri Lanka, 26-27 November 2010.

Sable M G and Rana D K, 2011, Study on antibiosis mechanism of resistance to $\mathrm{BPH}$ Nilaparvata lugens (Stal.) on resistant rice genotypes. Journal of Soils and Crops, 21(2): 196-202.

Santhanalakshi S, Saikumar S, Shubhada R, Sai Harini A, Pawan K, Shashidhar H E and Kadirves P, 2010, Mapping genetic locus linked to brown plant hopper resistance in rice Oryza sativa L. International Journal of Plant Breeding and Genetics, 4: 13-22.

Saxena R R and Saxena R R, 2009, Evaluation of rice germplasm against brown plant hopper. Rice Research, 2(1): 28-32.

Shoukat A R and Pandey I D, 2016, Molecular breeding for brown plant hopper in rice, Third International Conference on New Frontiers in Biotechnology, Science, Health and Medicine.

Udayasree M, Rajanikanth P, Varma N R G and Sreedhar M, 2018, Screening of selected rice genotypes for their resistance against brown planthopper, Nilaparvata lugens (Stal). International Journal of Current Microbiology and Applied Sciences, 7(11): 3138-3143.

Varma S K, Sable M G, Rana D K and Dubey V $\mathrm{K}$, 2014, Evaluation of different rice genotypes for BPH, Nilaparvata lugens (Stal.) resistance. International Journal of Plant Protection, 7(1):55-58.

Venugopala Reddy and Kalode M B, 1985, Mechanism of resistance in rice varieties showing differential reaction to brown planthopper. Proceedings of Indian Academy of Science (Anim. Sci.), 94(1): 3748.

Yong F Q, Ling C, Ping Z, Fang L and Rong B L, 2012, Identification of antixenosis and antibiosis in two newly explored brown planthopper-resistance rice lines. Advance Journal of Food Science and Technology, 4(5): 299-303.

Yongfu Q, Jianping G, Shengli J, Ming T, Lili Z and Guangcun He, 2011, Identification of antibiosis and tolerance in rice varieties carrying brown planthopper resistance genes. The Entomologia Experimentalis Applicata, 141: 224-231.

\section{How to cite this article:}

Farzanakorabu, B. Kisan, I. Shankergoud, Ayyangowda Patil, Mahantesh Shivyoggaya and Sujayhurali. 2020. Comparative Studies of Different Methods of Screening for Brown Plant Hopper (Nilaparvata lugens L.) Resistance in Rice Genotypes. Int.J.Curr.Microbiol.App.Sci. 9(06): 1914-1922. doi: https://doi.org/10.20546/ijcmas.2020.906.237 